\title{
THE LIKELY ANTECEDENTS OF IMPROBABLE EVENTS: OPTIMAL SEARCH STRATEGIES
}

\author{
A. M. FINK ${ }^{\prime}$ and L. BASS ${ }^{2}$ \\ (Received 25 November 1990; revised 5 September 1991)

\begin{abstract}
We use the "Brownian Bridge" of Schrödinger to model a statistical search problem in which the initial and final distributions of a random motion are given. We raise the question of how to use this information to optimally reconstruct a likely past event.
\end{abstract}

\section{Introduction}

Any non-negative solution of the diffusion equation

$$
u_{t}=D \nabla^{2} u
$$

with $D>0$ being the diffusion constant, determines a time-dependent probability density for the position of a single diffusing particle, given an initial probability density. Thus, in one-dimensional diffusion, a particle starting from $x=0$ at $t=0$ has the probability $u d x$ of position in the interval $(x, x+d x)$ at any later time $t>0$ given by the fundamental solution of (1.1)

$$
u(x, t)=g(x, t)=(4 \pi D t)^{-1 / 2} e^{-x^{2} / 4 D t}
$$

In contrast, when initial and final probability densities are given at time $t_{0}, t_{1}$, the probability density for locating the particle at an intermediate time $t\left(t_{0}<t<t_{1}\right)$ is not a solution of $(1.1)$, but is deduced from it as a nonlinear combination of solutions. The deduction hinges on the rule of multiplying probabilities of independent events occurring in conjunction, as follows.

\footnotetext{
'Permanent address: Mathematics Department, Iowa State University, Ames, IA 50011, USA.

${ }^{2}$ Mathematics Department, University of Queensland, Australia.

(C) Copyright Australian Mathematical Society 1992, Serial-fee code 0334-2700/92
} 
A particle starts one-dimensional diffusion at position $y$ at time $t_{0}$ and is found again at position $z$ and time $t_{1}$. What is the probability density $p\left(x, t \mid y, t_{0} ; z, t_{1}\right)$ of it having been at $x$ at time $t$ ? Using (1.2) with shifted origins of position and time, we see that the probability of diffusing from $y$ at $t_{0}$ to $z$ at $t_{1}$ via $x$ at $t$ :

$$
\begin{aligned}
& g\left(x-y, t-t_{0}\right) d x g\left(z-x, t_{1}-t\right) d z \\
& \quad=g\left(z-y, t_{1}-t_{0}\right) d z p\left(x, t \mid y, t_{0} ; z, t_{1}\right) d x,
\end{aligned}
$$

and hence

$$
p\left(x, t \mid y, t_{0} ; z, t_{1}\right)=\frac{g\left(x-y, t-t_{0}\right) g\left(z-x, t_{1}-t\right)}{g\left(z-y, t_{1}-t_{0}\right)} .
$$

A different (frequentist) derivation is given by Schrödinger [1].

The final event $\left(z, t_{1}\right)$ may be a very unlikely outcome of diffusion from position $y$ at $t_{0}$ (as assessed by $g\left(z-y, t_{1}-t_{0}\right)$ from (1.2)), but this outcome is a given fact to be included in the evaluation of $p\left(x, t \mid y, t_{0} ; z, t_{1}\right)$. In the relation between events $\left(y, t_{0}\right)$ and $\left(z, t_{1}\right)$ the validity of $(1.1)$ is thus suspended, while in the calculation of $p$ it is restored. Such liberties are permissible only because of the probabilistic interpretation of the solutions of (1.1). In contrast, heat conduction obeys (1.1) and (1.2) but provides no formal counterpart to (1.3). Whereas the Schrödinger equation differs from (1.1), the analogous construction of location probabilities which are bilinear in the solutions of the equation had motivated Schrödinger [1] to revive and develop Smoluchowski's consideration relating to (1.3). (See [1] for references.) Schrödinger's quantum considerations [1] related at first only to the reconstruction of past events, not to prediction of future events. For a recent review of this and subsequent quantum aspects, which do not concern us here, see [2, Ch. 7, pp. 258-260]. In the present paper we start from Schrödinger's mathematical formulations [1], but we keep to an interpretation in terms of classical probabilities for a macroscopic body in random walk, which leaves traces that are subject to searches calling for optimal strategies.

We shall be especially interested in optimal search strategies commencing after the completion of the diffusion process (after time $t_{1}$ ). The simplest case is illustrated by supposing that the event $\left(y, t_{0}\right)$ was a bank robbery by Jesse James (J.J.), the event $\left(z, t_{1}\right)$ was the capture of J.J. carrying no gold, and $(x, t)$ was the (hypothetical) event of J.J. burying the gold. If the value of the gold warrants only a finite area to be searched, how is that area to be selected?

In this problem and its generalisations, we assume that the probability density of the gold having been buried at $x$ at any time between $t_{0}$ and $t_{1}$ 
is the marginal probability density

$$
q\left(x \mid y, t_{0} ; z, t_{1}\right)=\frac{1}{t_{1}-t_{0}} \int_{t_{0}}^{t_{1}} p\left(x, t \mid y, t_{0} ; z, t_{1}\right) d t,
$$

proportional to the time-average of the location probability (1.3) at each $x$. The assumption that the robbers evaded pursuit by random walks is surprisingly well attested [3]:

"The James-Younger gang meandered the woodlands of Rice, Waseca, and Blue Earth counties for a week, sometimes recrossing the same ravine four times in their windings, or clocking a village so that stores and hovels shied from at noon were confronted a second time at two. With Bill Chadwell gone, they were gloomily lost in green woods or limited by foreign creeks and rivers that were too deep to ford. Even as late as four nights after they'd run out of Northfield, the bedraggled gang was spied by boys who lived no more than fifteen miles away. At a small hotel near Shieldsville a posse of ten from Faribault was eating supper when the gang rode up to water their horses and became perplexed by the great variety of shotguns and rifles angled against the hotel's porch railing. Jesse crept up to the porch and pressed against the screen, peering in. The posse men stopped talking or chewing or lifting their spoons and looked at Jesse with apprehension or stupidity, perceiving at once who he was; and they sheepishly permitted him to jump down and sprint away with his gang before they got up from their suppers."

It will be apparent that the Jesse James metaphor stands for a number of important diffusion processes such as the spread of an epidemic by a randomly moving infectious agent.

Once movement by diffusion is postulated, the question arises as to the magnitude of the diffusion constant. One possibility is to assume that it is known, another is to estimate it as $(y-z)^{2} /\left(t_{1}-t_{0}\right)$. That estimate is, however, in conflict with the suspension of the validity of (1.1) in relating the events $\left(y, t_{0}\right)$ and $\left(z, t_{1}\right)$ (see above). This criticism is emphasised in our generalisation of the search problem arising from an intermediate sighting of J.J. at some (third) place and time. The resulting estimates of $D$ may be so different from each other that the best strategy may be that which is least dependent on the value of $D$.

The problem of good estimates for $D$ is an important one in the statistical literature and will not be discussed here. Strategies which may be insensitive to $D$ are developed below.

A further generalisation of the simplest problem is the case of the final event $\left(z, t_{1}\right)$ being replaced by a probability density at $t_{1}$. Should the search then be guided by an optimal strategy based on this information or would a better strategy expend some effort to first narrow the probability density 
distribution at $t_{1}$ ? When $\left(y, t_{0}\right)$ is also replaced by a probability distribution (at $t_{0}$ ), the determining equations found in Schrödinger [1] are complicated (see Appendix 3).

The main results are arranged in the body of the paper and its appendices as follows: in Section 2, we discuss the one-dimensional problem with simple sightings. The details of the calculations are given in Appendix 1. In Section 3 , we do the extended problem with a final probability measure. In addition, Appendices 2, 3, and 4 give details in the two dimensional case and the centre of mass argument.

\section{One-dimensional problem}

We have formulated a search problem in which we have a continuous function $q(x)$ defined on $\mathbb{R}^{n}$ such that $0 \leq q(x)$. It is

$$
\max _{V(\mathbf{R})=a} \int_{\mathbb{R}} q(x) d x
$$

where $V$ is the volume function and $\mathbb{R}$ is a measurable set with $a$ a given fixed number.

We let $\mathbb{R}_{d}=\{x \mid q(x) \geq d\}$ and $v(d)=V\left(\mathbb{R}_{d}\right)$. Then $v(0)=+\infty, v$ is non-increasing, $v(d) \rightarrow 0$ for $d \rightarrow \infty$. It is continuous from the left but may have jump discontinuities due to flat spots on the graph of $q$. The primitive search theorem is easy to prove.

TheOREM 1. Let $d_{0}=\sup \left\{d \mid v\left(\mathbb{R}_{d}\right) \geq a\right\}$. Then any subset $\mathbb{R} \subset \mathbb{R}_{d_{0}}$ such that $\mathbb{R} \supset\left\{x \mid q(x)>d_{0}\right\}$ solves the maximisation problem (I).

Proof. Let $\mathbb{R}$ be a given measurable set whose volume is $a$. Define $\mathbb{R}_{1}=$ $\mathbb{R} \cap \mathbb{R}_{d_{0}}$ and $\mathbb{R}_{2}=\mathbb{R} \cap \mathbb{R}_{d_{0}}^{c}$. Then $V\left(\mathbb{R}_{2}\right)=a-V\left(\mathbb{R}_{1}\right)$ and by the choice of $d_{0}, V\left(\mathbb{R}_{d_{0}} \backslash \mathbb{R}_{1}\right) \geq a-V\left(\mathbb{R}_{1}\right)=V\left(\mathbb{R}_{2}\right)$. Consequently, there is a measurable subset $\mathbb{R}_{3} \subset \mathbb{R}_{d_{0}} \backslash \mathbb{R}_{1}$ such that $V\left(\mathbb{R}_{3}\right)=V\left(\mathbb{R}_{2}\right)$. If $x \in \mathbb{R}_{2}$ and $y \in \mathbb{R}_{3}$ then $q(x) \leq d_{0} \leq q(y)$ and

$$
\int_{\mathbf{R}_{2}} q(x) d x \leq d_{0} V\left(\mathbb{R}_{2}\right)=d_{0} V\left(\mathbb{R}_{3}\right) \leq \int_{\mathbf{R}_{3}} q(y) d y .
$$

Hence $\int_{\mathbb{R}_{1} \cup \mathbb{R}_{3}} q(x) d x \geq \int_{\mathbb{R}} q(x) d x$ and $V\left(\mathbb{R}_{1} \cup \mathbb{R}_{3}\right)=a$. We have shown that for every set $\mathbb{R}$ with $V(\mathbb{R})=a$, there is a subset of $\mathbb{R}_{d_{0}}$ that majorises $\int_{\mathbb{R}} q(x) d x$. If $v\left(\mathbb{R}_{d_{0}}\right)=a$ we are done. In the contrary case, $v\left(\mathbb{R}_{d_{0}}\right)>a$ while $\lim _{d \rightarrow d_{0}^{+}} v\left(\mathbb{R}_{d}\right)<a$. This means that $V\left\{x \mid q(x)=d_{0}\right\}>0$, and 
$v\left\{x \mid q(x)>d_{0}\right\}<a$. Then any set $\mathbb{R}$ such that $\left\{x \mid q(x)>d_{0}\right\} \subset \mathbb{R} \subset \mathbb{R}_{d_{0}}$ with $V(\mathbb{R})=a$ solves the problem.

For the one-dimensional problem, we show in Appendix 1 that $q\left(x \mid y, t_{0} ; z, t_{1}\right)$ is symmetric about $x=(y+z) / 2$, constant on the interval between $y$ and $z$ and decreasing to zero on the suburbs of the interval with end points $y$ and $z$. It follows from Theorem 1, that for every $a$ we may take an interval centered at $z+y / 2$. We state this formally.

Corollary 1. For one-dimensional search the optimal strategy for problem I is to take an interval $\left[\frac{y+z-a}{2}, \frac{y+z+a}{2}\right]$.

It is interesting to note that the parameter $D$ does not enter into this result. It is also interesting to see what the flat-topped behavior adds to the discussion of the problem. From (4.12) in Appendix 1, we see that $(y<z)$

$$
q\left(x \mid y, t_{0} ; z, t_{1}\right)=\frac{2 \sqrt{\lambda} e^{\lambda}}{|y-z|} \int_{\sqrt{\lambda}}^{\infty} e^{-s^{2}} d s \quad \text { on }[y, z],
$$

where $\lambda=(y-z)^{2} / 4 D\left(t_{1}-t_{0}\right)$. We are interested in the portion of $q$ that is in the tails outside $[y, z]$. Since $\int_{-\infty}^{\infty} q(x) d x=1$, we get some idea of the tails by computing $\int_{y}^{z} q\left(x \mid y, t_{0} ; z, t_{1}\right) d x \equiv k(\lambda)$. We see that

$$
k(\lambda)=2 \sqrt{\lambda} e^{\lambda} \int_{\lambda}^{\infty} e^{-s^{2}} d s .
$$

An alternate formula is given in Lemma (2) of Appendix 1,

$$
k(\lambda)=2\left(\frac{\lambda}{\pi}\right)^{1 / 2} \int_{0}^{\infty} \frac{e^{-\lambda v^{2}}}{1+v^{2}} d v .
$$

In (2.2) we change variables to get

$$
k(\lambda)=\frac{2}{\sqrt{\pi}} \int_{0}^{\infty} \frac{e^{-u^{2}}}{1+u^{2} / \lambda} d u .
$$

From this latter formula, we see that $k^{\prime}(\lambda)>0, k^{\prime \prime}(\lambda)<0$ and $k(\infty)=1$. From (2.1) we see that $k(0)=0$ and $k^{\prime}(0)=\lim _{\lambda \rightarrow 0^{+}} k(\lambda) / \lambda=+\infty$. Also we see the influence of the diffusion coefficient $D$. For $y$ and $z$ fixed, $\lambda D=(y-z)^{2} / 4\left(t_{1}-t_{0}\right)$ so that $D$ large corresponds to $k$ being small, and $D$ small to $k$ almost 1 .

The tail of

$$
q\left(x \mid y, t_{0} ; z, t_{1}\right)=\frac{2 \sqrt{\lambda} e^{\lambda}}{|y-z|} \int_{\sqrt{\lambda}|2 x-y-z| /|y-z|}^{\infty} e^{-s^{2}} d s,
$$


so that for a fixed $\lambda$ this drops off to zero rapidly as $|x|$ increases. In fact

$$
\int_{v}^{\infty} e^{-s^{2}} d s \sim \frac{e^{-v^{2}}}{2 v}
$$

More precise bounds for Mill's ratio may be found in Mitrinović [4, page 179].

We now turn to a different scenario. Suppose that there was an intermediate sighting, that is we know the location at $\left(y, t_{0}\right),\left(z, t_{1}\right)$ and $\left(w, t_{2}\right)$ with $t_{0}<t_{1}<t_{2}$. Then we want to write $q\left(x \mid y, t_{0} ; z, t_{1} ; w, t_{2}\right)$. We use the principle of independent increments so that on the interval $\left(t_{0}, t_{1}\right)$ the knowledge $\left(w, t_{2}\right)$ adds no information and on the interval $\left(t_{1}, t_{2}\right)$ the knowledge of $\left(y, t_{0}\right)$ is superfluous. It follows that

$$
p\left(x, t \mid y, t_{0} ; z, t_{1} ; w, t_{2}\right)= \begin{cases}p\left(x, t \mid y, t_{0} ; z, t_{1}\right), & t \in\left(t_{0}, t_{1}\right), \\ p\left(x, t \mid z, t_{1} ; w, t_{2}\right), & t \in\left(t_{1}, t_{2}\right) .\end{cases}
$$

Thus

$$
\begin{aligned}
q\left(x \mid y, t_{0} ; z, t_{1} ; w, t_{1}\right)= & \frac{1}{t_{2}-t_{0}} \int_{t_{0}}^{t_{1}} p\left(x, t \mid y, t_{0} ; z, t_{1}\right) d t \\
& +\frac{1}{t_{2}-t_{0}} \int_{t_{1}}^{t_{2}} p\left(x, t \mid y, t_{1} ; z, t_{2}\right) d t \\
= & \frac{t_{1}-t_{0}}{t_{2}-t_{0}} q\left(x \mid y, t_{0} ; z, t_{1}\right) \\
& +\frac{t_{2}-t_{1}}{t_{2}-t_{0}} q\left(x \mid z, t_{1} ; w, t_{2}\right) .
\end{aligned}
$$

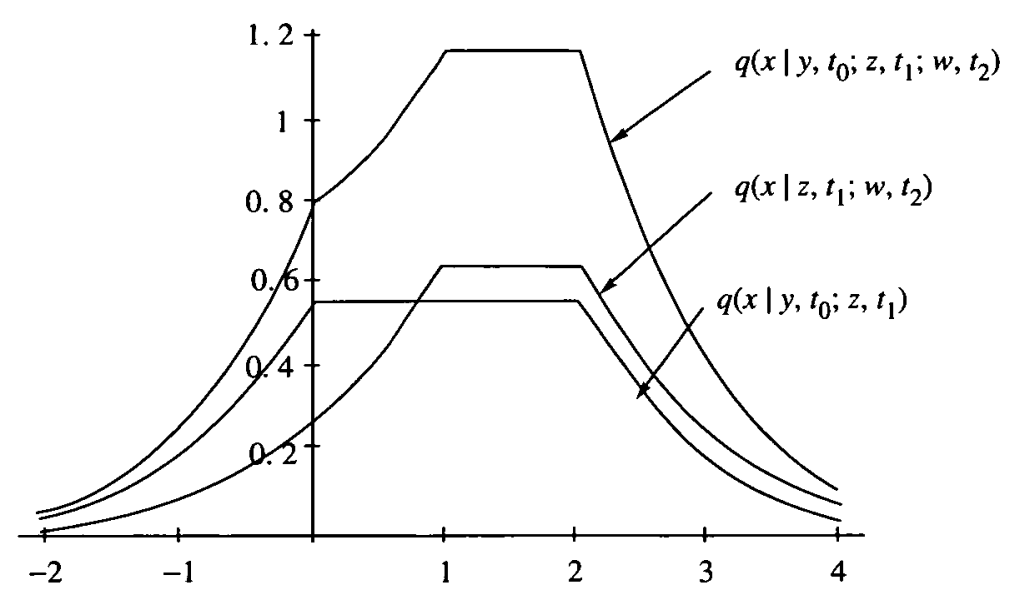

Figure 1. 


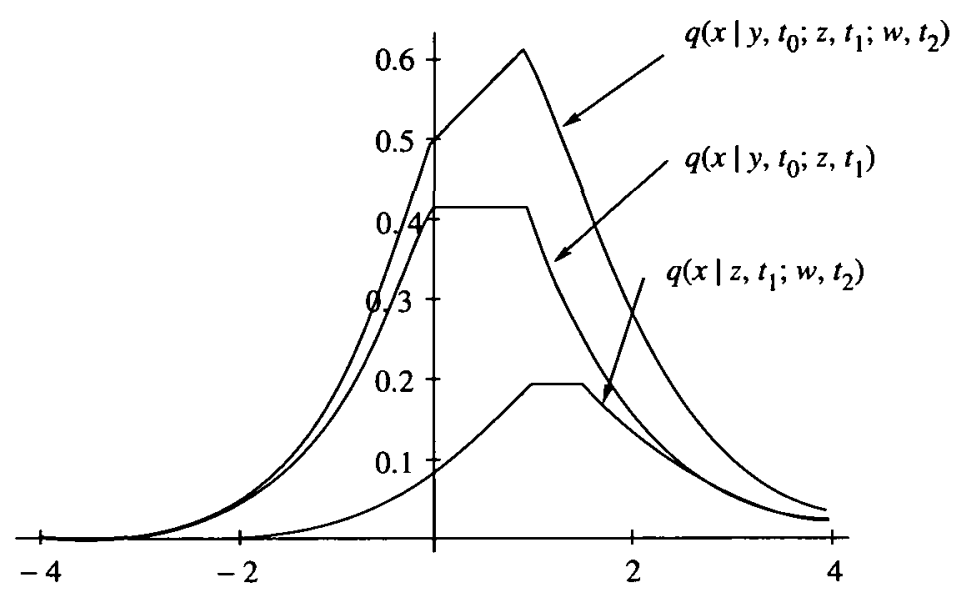

Figure 2.

If the arrangement of spatial points is $y<w<z$ then Figure 1 applies, so that our primitive search theorem gives an obvious optimal search strategy if $a<|z-w|$.

On the other hand, if the arrangement is $y<z<w$ then Figure 2 applies. In this case more detailed information is necessary for any optimal search. In particular, we need to have an estimate for the diffusion constant $D$. In fact we have two obvious estimates for $D, E(z-y)^{2}=2 D\left(t_{1}-t_{0}\right)$ and $E(w-z)^{2}=2 D\left(t_{2}-t_{1}\right)$ which may vary widely.

\section{Extended problem}

We now extend our discussion to a situation where the sighting at $t_{1}$ is not certain, that is, the observation at $t_{1}$ is distributed with a distribution function $\omega(z)$.

Suppose, by way of argument, that $N$ particles were sent from $y$ at time $t_{0}$ and were observed at $t_{1}$ according to the density $\omega(z)$. Consider an elementary "patch" $\Delta_{i}$ with $\int_{\Delta_{i}} d \omega(z)$ small, and $z_{i} \in \Delta_{i}$. Then the number of particles seen at $z_{i}$ at time $t_{1}$ is approximately $N \int_{\Delta_{i}} d \omega(z)$. Hence the total number at $(x, t)$ is given by the sum over disjoint patches and is

$$
N \int_{\mathbf{R}} p\left(x, t \mid y, t_{0} ; z, t_{1}\right) d \omega(z)
$$


Consequently, if $p\left(x, t \mid y, t_{0} ; \omega, t_{1}\right)$ is the probability density of the diffusion beginning at $\left(y, t_{0}\right)$ and observed at $t_{1}$ with distribution $\omega$, then

$$
p\left(x, t \mid y, t_{0} ; \omega, t_{1}\right)=\int_{\mathbf{R}} p\left(x, t \mid y, t_{0} ; z, t_{1}\right) d \omega(z),
$$

and by Fubini's theorem, the average time spent at $x$ is

$$
q\left(x \mid y, t_{0} ; \omega, t_{1}\right)=\int_{\mathbf{R}} q\left(x \mid y, t_{0} ; z, t_{1}\right) d \omega(z) .
$$

It seems intuitively clear that $q\left(x \mid y, t_{0} ; \omega, t_{1}\right)$ becomes more peaked when $d \omega$ is more concentrated. In view of this guess, we formulate a new search problem in the face of the uncertain facts $d \omega$. Loosely speaking, we choose to spend some energy in searching in the support of $d \omega$ in order to make it more concentrated, but then we can search for the gold only in a smaller region. The two desires have opposing effects and so we have a true optimisation problem.

To be more precise, suppose $\left(y, t_{0}\right)$ and $\left(\omega, t_{1}\right)$ are given along with a number $a$ as the volume of search we may conduct. We may take a number $b$ with $0 \leq b \leq a$ and search an area $B$ in support of $d \omega$. If we find J.J. in $B$ then instead of using $q\left(x \mid y, t_{0} ; w, t_{1}\right)$ we use instead $q\left(x \mid y, t_{0} ; \omega_{B}, t_{1}\right)$, where $d \omega_{B}=d \omega \chi_{B} / \int_{B} d \omega$ with $\chi_{B}$ the indicator function of $B$.

We would then use set $\mathbb{R}_{d}$ of volume $a-b$ to search for the gold. We are now maximising the expected value. Suppose for example, that we search $B$ and find $z \in B$. We use

$$
\begin{aligned}
q\left(x \mid y, t_{0} ; \omega_{B}, t_{1}\right) & =\int_{\mathbb{R}} q\left(x \mid y, t_{0} ; z, t_{1}\right) d \omega_{B}(z) \\
& =\int_{B} q\left(x \mid y, t_{0} ; z, t_{1}\right) d \omega(z) / \int_{B} d \omega(z)
\end{aligned}
$$

with probability $\int_{B} d \omega(z)$. Hence, the expected value $q_{1}$ of finding the gold is the integral with respect to $x$ of $q\left(x \mid y, t_{0} ; \omega_{B}, t_{1}\right)$ over an upper set of volume $a-b$ times the probability $\int_{B} d \omega(z)$ of this procedure. We get

$$
q_{1}(B)=\int_{\mathbf{R}_{d_{0}}}\left(\int_{B} q\left(x \mid y, t_{0} ; z, t_{1}\right) d \omega(z)\right) d x
$$

with $\mathbb{R}_{d_{0}}$ an upperset of $\int_{B} q\left(x \mid y, t_{0} ; z, t_{1}\right) d \omega(z), v\left(\mathbb{R}_{d_{0}}\right)=a-b$.

If we do not find $z \in B$, then this is tantamount to finding $z \in B^{c}$ so that we get an expected value $q_{2}$

$$
q_{2}(B)=\int_{\mathbf{R}_{d_{0}}^{\prime}}\left(\int_{B^{c}} q\left(x \mid y, t_{0} ; z, t_{1}\right) d \omega(z)\right) d x
$$

with $\mathbb{R}_{d_{0}}^{\prime}$ an upperset of $\int_{B^{c}} q\left(x \mid y, t_{0} ; z, t_{1}\right) d \omega(z), v\left(\mathbb{R}_{d_{0}}^{\prime}\right)=a-b$. 
Our new problem is

$$
\max _{\substack{b, B \\ v(B)=b \\ 0 \leq b \leq a}}\left[q_{1}(B)+q_{2}(B)\right]
$$

There actually are two versions of this problem. We may assume that the diffusion parameter $D$ is fixed, or we can agree to estimate it. In the latter case, presumably we would have different values of $D$ in the computations of $q_{1}$ and $q_{2}$, while in the former case we would use the same.

As a way of understanding this problem we offer two examples.

EXAMPle 1. Let $d \omega(z)=\frac{1}{2} \delta_{-1}(z)+\frac{1}{2} \delta_{1}(z)$ with $\left(y, t_{0}\right)=(0,0)$ where $\delta_{x}(z)$ is a point mass at $x$. Let $a<1$. If one devotes $\varepsilon$ to search at -1 , then with probability $1 / 2$, one integrates $q\left(x \mid 0,0 ;-1, t_{1}\right)$ over an interval of length $a-\varepsilon$ where this $q$ is a constant. If one does not find J.J. at -1 then one integrates $q\left(x \mid 0,0 ; 1, t_{1}\right)$ over an interval where this is a constant. But these two are the same so the expected value is $(a-\varepsilon) q^{*}, q^{*}$ the constant value of $q\left(x \mid 0,0 ; 1, t_{1}\right)$ on $[0,1]$. On the other hand, if no search at \pm 1 is conducted, then one integrates $\frac{1}{2} q\left(x \mid 0,0 ;-1, t_{1}\right)+\frac{1}{2} q\left(x \mid 0,0 ; 1, t_{1}\right)=$ $\bar{q}(x)$ over an interval $[-a, a]$. But $\bar{q}<q^{*}$ except at zero where the two are equal. For small $\varepsilon$, the search procedure is optimal. Note that for all three cases, searching at -1 and finding, searching at 1 and finding, and not searching, the expected value of $E(z-y)^{2}=1$ so that one could logically pick $2 D t_{1}=1$ so that the second version of our problem is not different from the first.

As a preliminary to doing a version of the second problem we note that the formula for $q$ may be written in a form to show the explicit dependence on $D$. If we let $\lambda=(y-z)^{2} / \beta$ and $\beta=4 D\left(t_{1}-t_{0}\right)$ then (4.6) of Appendix 1 becomes

$$
q\left(x \mid y, t_{0} ; z, t_{1}\right)=\frac{2}{\sqrt{\pi}}|y-z| \int_{0}^{\infty} \frac{e^{-u^{2}} d u}{(y-z)^{2}+\beta u^{2}}
$$

for $x$ between $y$ and $z$. If we take $y=0$ and $d \omega(z)$ to be a uniform distribution on $[b, c], b>0$ then for $0 \leq x \leq b$ we have by integration of (3.5)

$$
q\left(x \mid 0, t_{0} ; \omega, t_{1}\right)=\frac{1}{(c-b) \sqrt{\pi}} \int_{0}^{\infty} \ln \left(\frac{c^{2}+\beta u^{2}}{b^{2}+\beta u^{2}}\right) e^{-u^{2}} d u .
$$

EXAMPLE 2. We take $d \omega(z)$ to be the uniform distribution on $[1,2]$ and $a \leq 1$. First we fix $D$. If one does not search, then one integrates (the 
constant) $q\left(x \mid 0, t_{0} ; \omega, t_{1}\right)$ over an interval of length $a$, to arrive at

$$
\bar{q}=\frac{a}{\sqrt{\pi}} \int_{0}^{\infty} \ln \left(\frac{4+\beta u^{2}}{1+\beta u^{2}}\right) e^{-u^{2}} d u .
$$

On the other hand, if we choose to search an interval $[b, b+r], 1 \leq b \leq$ $b+r \leq z$, and we find J.J., then we get with probability $r$,

$$
\bar{q}=\frac{a-r}{r \sqrt{\pi}} \int_{0}^{\infty} \ln \left(\frac{(b+r)^{2}+\beta u^{2}}{b^{2}+\beta u^{2}}\right) e^{-u^{2}} d u .
$$

If we do not find, then with probability $(1-r)$ we get

$$
\bar{q}_{2}=\frac{a-r}{(1-r) \sqrt{\pi}} \int_{0}^{\infty}\left\{\ln \left(\frac{b^{2}+\beta u^{2}}{1+\beta u^{2}}\right)+\ln \left(\frac{4+\beta u^{2}}{(b+r)^{2}+\beta u^{2}}\right)\right\} e^{-u^{2}} d u .
$$

The expected value is therefore $\bar{q}=r \bar{q}_{1}+(1-r) \bar{q}_{2}$,

$$
\bar{q}=\frac{a-r}{\sqrt{\pi}} \int_{0}^{\infty} \ln \left[\frac{4+\beta u^{2}}{1+\beta u^{2}}\right] e^{-u^{2}} d u=\frac{(a-r)}{a} \bar{q} .
$$

This is clearly maximised by taking $r=0$, that is, do not search for J.J.

This calculation is an instance of a theorem.

Theorem 2. Let $D$ be fixed, $y=0$, and $0<a \leq C$. Suppose $d \omega(z)$ has support $\subset(C, \infty)$, then the best strategy is not to search for J.J.

Proof. As above, for all instances of search, we are integrating $q$ over an interval $\subset[0, C]$ over which $q$ is a constant. That is in (3.3) and (3.4) $R_{d_{0}}$ and $R_{d_{0}}^{1}$ may be taken to be the same. It follows that $q_{1}(B)+q_{2}(B)=$ $(a-b) \int_{0}^{\infty} q\left(x \mid y, t_{0} ; z, t_{1}\right) d \omega(z)$, which is maximised by taking $b=0$.

We return to a discussion of Example 2. If we now take a version of the problem that we estimate $D$, then suppose we use $E\left(z^{2}\right)=2 D\left(t_{1}-t_{0}\right)$. In (3.7) we would take $2 D\left(t_{1}-t_{0}\right)=\int_{1}^{2} z^{2} d z=\frac{7}{3}$ so that $\beta=\frac{14}{3}$. In (3.8) we take $\beta_{1}=2 \int_{b}^{b+r} z^{2} d z=\frac{2}{3}\left[(b+r)^{3}-b^{3}\right]$ while in (3.9) we take $\beta_{2}=\frac{2}{3}\left[b^{3}+7-(b+r)^{3}\right]$. The formula for $\bar{q}$ becomes

$$
\bar{q}=\frac{a-r}{\sqrt{\pi}} \int_{0}^{\infty} e^{-u^{2}} \ln \left[\frac{(b+r)^{2}+\beta_{1} u^{2}}{b^{2}+\beta_{1} u^{2}} \frac{b^{2}+\beta_{2} u^{2}}{1+\beta_{2} u^{2}} \frac{4+\beta_{2} u^{2}}{(b+r)^{2}+\beta_{2} u^{2}}\right] d u .
$$


This has to be compared to $\bar{q}$ with $\beta=14 / 3$, a rather formidable problem.

\section{Appendix 1}

We compute the formulae for $q\left(x \mid y, t_{0} ; z, t_{1}\right)$ here starting with (1.4) which we repeat.

$$
q\left(x \mid y, t_{0} ; z, t_{1}\right)=\frac{1}{t_{1}-t_{0}} \int_{t_{0}}^{t_{1}} \sqrt{\frac{t_{1}-t_{0}}{\left(t-t_{0}\right)\left(t_{1}-t\right)}} \frac{1}{\sqrt{4 \pi D}} e^{-I / 4 D} d t
$$

where

$$
I=\frac{(x-y)^{2}}{t-t_{0}}+\frac{(z-x)^{2}}{t_{1}-t}-\frac{(z-y)^{2}}{t_{1}-t_{0}} .
$$

The change of the variables $t=s t_{1}+(1-s) t_{0}$ leads to

$$
q\left(x \mid y, t_{0} ; z, t_{1}\right)=\frac{1}{\sqrt{4 \pi D\left(t_{1}-t_{0}\right)}} \int_{0}^{1} \frac{d s}{\sqrt{s(1-s)}} \exp \left(-\frac{[x-s z-y(1-s)]^{2}}{4 D\left(t_{1}-t_{0}\right) s(1-s)}\right) .
$$

We simplify this expression by setting

$$
4 D\left(t_{1}-t_{0}\right)=(y-z)^{2} \lambda^{-1}
$$

and

$$
\frac{x-z}{y-z}=\frac{1}{2}-u
$$

We obtain

$$
q\left(x \mid y, t_{0} ; z, t_{1}\right)=\left(\frac{\lambda}{\pi}\right)^{\frac{1}{2}}|y-z|^{-1} \int_{0}^{1} \exp \left\{-\lambda \frac{\left(\frac{1}{2}+u-s\right)^{2}}{s(1-s)}\right\} \frac{d s}{\sqrt{s(1-s)}} .
$$

Call this expression $I(u)$.

LEMMA 1. The integral in (4.5) is even and decreases for $u \geq 1 / 2$.

Proof. In $I(-u)$ replace the variable $s$ by $1-s$ to see that $I(-u)=I(u)$. If $u \geq 1 / 2$ then $1 / 2+u-s \geq 0$ for $0 \leq s \leq 1$. Then $u_{1}>u_{2} \geq 1 / 2$ implies that $\left(1 / 2+u_{1}-s\right)^{2} \geq\left(1 / 2+u_{2}-s\right)^{2}$ for $0 \leq s \leq 1$ (noting that $\lambda>0$ ) so $I\left(u_{1}\right) \leq I\left(u_{2}\right)$.

Lemma 2. For $|u| \leq 1 / 2, I(u)$ is a constant, in fact

$$
I(u)=\left(\frac{\lambda}{\pi}\right)^{\frac{1}{2}} 2|y-z|^{-1} \int_{0}^{\infty} \frac{e^{-\lambda v^{2}}}{1+v^{2}} d v .
$$


Proof. Since $I(u)$ is even we may restrict ourselves to $0 \leq u<1 / 2$. We set $s=\sin ^{2}(\theta / 2), 0 \leq \theta \leq \pi$ and then $\phi=\pi-\theta$ to compute

$$
I(u)=\left(\frac{\lambda}{\pi}\right)^{\frac{1}{2}}|y-z|^{-1} \int_{0}^{\pi} e^{-\lambda[2 u \csc \phi-\cot \phi]^{2}} d \phi .
$$

If $g(\phi)=2 u \csc \phi-\cot \phi$ then $g(0)=-\infty, g(\pi)=+\infty$ and $g^{\prime}(\phi)>0$ on $(0, \pi)$, so we may change variables by

$$
v=2 u \csc \phi-\cot \phi \text {. }
$$

If we set $\sin \alpha=v / \sqrt{1+v^{2}}$ and $\cos \alpha=1 / \sqrt{1+v^{2}}$ for $|\alpha|<\pi / 2$ we have from (4.8) that

$$
\cos (\phi-\alpha)=2 u / \sqrt{1+v^{2}}
$$

Since $\phi=0$ corresponds to $v=-\infty$ and $\alpha=-\pi / 2$, and $\phi=\pi$ corresponds to $v=+\infty$ and $\alpha=\pi / 2$ we get

$$
\phi=\alpha+\cos ^{-1}\left(2 u / \sqrt{1+v^{2}}\right)=\tan ^{-1} v+\cos ^{-1}\left(2 u / \sqrt{1+v^{2}}\right) .
$$

Thus

$$
I(u)=\left(\frac{\lambda}{\pi}\right)^{\frac{1}{2}}|y-z|^{-1} \int_{-\infty}^{\infty} \frac{e^{-\lambda v^{2}}}{1+v^{2}}\left[1+\frac{2 u v}{\sqrt{1+v^{2}-4 u^{2}}}\right] d v .
$$

Since the second term is odd and the first even we get (4.6). In (4.5) it is clear that $I(u)$ is a continuous function of $u$ so (4.6) holds also at $|u|=1 / 2$.

LeMma 3. For $|u|>1 / 2$,

$$
I(u)=2\left(\frac{\lambda}{\pi}\right)^{\frac{1}{2}}|y-z|^{-1} e^{\lambda} e^{-4 u^{2} \lambda} \int_{0}^{\infty} \frac{e^{-4 \lambda u^{2} s^{2}}}{1+s^{2}} d s .
$$

Proof. We again begin with (4.7) and consider $(2 u>1)$ the change of variable $v=g(\phi)$. Since $g^{\prime}(\phi)=0$ when $\cos \phi=(2 u)^{-1}$ we write $I(u)=$ $\int_{0}^{\cos ^{-1} u / 2}+\int_{\cos ^{-1} u / 2}^{\pi}$. As above, we get $\phi=0, \pi$ corresponding to $v=+\infty$ and $\alpha=\frac{\pi}{2}$ so that $\phi=\tan ^{-1} v-\cos ^{-1} 2 u / \sqrt{1+v^{2}}$ in the first integral and $\phi=\tan ^{-1} v+\cos ^{-1} 2 u / \sqrt{1+v^{2}}$ in the second integral. The integrals are over the same interval and their sum gives

$$
I(u)=\left(\frac{\lambda}{\pi}\right)^{\frac{1}{2}}|y-z|^{-1} \int_{v_{0}}^{\infty} \frac{4|u| v e^{-\lambda v^{2}} d v}{\left(1+v^{2}\right) \sqrt{1+v^{2}-4 u^{2}}}
$$

where $v_{0}=\sqrt{4 u^{2}-1}$. We finally make the change of variables $1+v^{2}=$ $4 u^{2}\left(1+s^{2}\right)$ to arrive at (4.11). The integrals in (4.6) and (4.11) are similar and we can rewrite them in terms of more familiar functions. 
Lemma 4. For $\lambda>0$,

$$
\int_{0}^{\infty} \frac{e^{-\lambda s^{2}}}{1+s^{2}} d s=\sqrt{\pi} e^{\lambda} \int_{\sqrt{\lambda}}^{\infty} e^{-s^{2}} d s
$$

Proof. Let $h(\lambda)=\int_{0}^{\infty} \frac{e^{-\lambda s^{2}}}{1+s^{2}} d s$. Then $h(0)=\frac{\pi}{2}$ and $h^{\prime}(\lambda)=h(\lambda)-$ $\int_{0}^{\infty} e^{-\lambda s^{2}} d s=h(\lambda)-\frac{\sqrt{\pi}}{2 \sqrt{\lambda}}$. We find that

$$
\begin{aligned}
h(\lambda) & =\frac{\pi}{2} e^{\lambda}-\frac{\sqrt{\pi}}{2} e^{\lambda} \int_{0}^{\lambda} \frac{e^{-t}}{\sqrt{t}} d t \\
& =e^{\lambda} \frac{\pi}{2}\left[1-\frac{2}{\sqrt{\pi}} \int_{0}^{\sqrt{\lambda}} e^{-s^{2}} d s\right]=e^{\lambda} \frac{\pi}{2}\left[\frac{2}{\sqrt{\pi}} \int_{\sqrt{\lambda}}^{\infty} e^{-s^{2}} d s\right] .
\end{aligned}
$$

\section{Proposition.}

$$
q\left(x \mid y, t_{0} ; z, t_{1}\right)=2 \sqrt{\lambda}|y-z|^{-1} e^{\lambda} \begin{cases}\int_{\sqrt{\lambda}}^{\infty} e^{-s^{2}} d s \quad x \text { between } y \text { and } z, \\ \int_{2 u \sqrt{\lambda}}^{\infty} E^{-s^{2}} d s & \text { otherwise, }\end{cases}
$$

with $\lambda=(y-z)^{2} / 4 D\left(t_{1}-t_{0}\right)$ and $4 u^{2}=\frac{(2 x-y-z)^{2}}{(y-z)^{2}}$. An alternate expression for the constant is $e^{\lambda} / \sqrt{D\left(t_{1}-t_{0}\right)}$.

\section{Appendix 2}

(The two-dimensional case). We have

$$
\begin{aligned}
q\left(x \mid y, t_{0} ; z, t_{1}\right) & =\frac{1}{t_{1}-t_{0}} \int_{t_{0}}^{t_{1}} p\left(x_{1}, t \mid y_{1}, t_{0} ; z_{1}, t_{1}\right) p\left(x_{2}, t \mid y_{2}, t_{0} ; z_{2}, t_{1}\right) d t \\
& =\frac{1}{4 \pi D\left(t_{1}-t_{0}\right)} \int_{t_{0}}^{t_{1}} e^{-\frac{1}{4 t_{1} I_{1}}} e^{-\frac{1}{4 b_{2} I_{2}}} \frac{d t}{\left(t-t_{0}\right)\left(t_{1}-t\right)}
\end{aligned}
$$

where

and

$$
D=\sqrt{D_{1} D_{2}}
$$

$$
I_{i}=\frac{\left(x_{i}-y_{i}\right)^{2}}{t-t_{0}}+\frac{\left(z_{i}-x_{i}\right)^{2}}{t_{1}-t}-\frac{\left(z_{i}-y_{i}\right)^{2}}{t_{1}-t_{0}} .
$$

With $t=s t_{1}+(1-s) t_{0}$ as in Appendix 1 , and

$$
\frac{x_{i}-z_{i}}{y_{i}-z_{i}}=\frac{1}{2}-u_{i}, \quad 4 D_{i}\left(t_{1}-t_{0}\right)=\left(y_{i}-z_{i}\right)^{2} \lambda_{i}^{-1}
$$


we arrive at

$$
\begin{aligned}
q\left(x \mid y, t_{0} ; z, t_{1}\right)= & \frac{1}{4 \pi D\left(t_{1}-t_{0}\right)} \\
& \cdot \int_{0}^{1} \exp \left\{\frac{-\lambda_{1}\left[\frac{1}{2}+u_{1}-s\right]^{2}-\lambda_{2}\left[\frac{1}{2}+u_{2}-s\right]^{2}}{s(1-s)}\right\} \frac{d s}{s(1-s)} .
\end{aligned}
$$

Again we make the change of variable, $s=\sin ^{2} \phi / 2,0 \leq \phi \leq \pi$, to obtain

$$
q\left(x \mid y, t_{0} ; z, t_{1}\right)=\frac{e^{\lambda_{1}+\lambda_{2}}}{2 \pi D\left(t_{1}-t_{0}\right)} \int_{0}^{\pi} \csc \phi e^{-\left(A \csc ^{2} \phi+B \csc \phi \cot \phi\right)} d \phi
$$

with

$$
\left\{\begin{array}{l}
A=\lambda_{1}\left(1+4 u_{1}^{2}\right)+\lambda_{2}\left(1+4 u_{2}^{2}\right) \\
B=4 u_{1} \lambda_{1}+4 u_{2} \lambda_{2}
\end{array}\right.
$$

We assume $B>0$, with $B=0$ treated by continuity. We further note that $A-B=\lambda_{1}\left(1-2 u_{1}\right)^{2}+\lambda_{2}\left(1-2 u_{2}\right)^{2}>0$ unless $u_{1}=u_{2}=1 / 2$ which corresponds to $x=z$. We exclude this case and proceed to make a change of variables

$$
v=g(\phi)=\csc ^{2} \phi(A+B \cos \phi) .
$$

Then $\phi=0, \pi$ correspond to $v=+\infty$ and $\phi_{0}$ to $v_{0}$ where $g^{\prime}\left(\phi_{0}\right)=0$ determines $\phi_{0}$ and is

$$
2 A \cos \phi_{0}+B \cos ^{2} \phi_{0}+B=0 .
$$

The polynomial $2 A x+B x^{2}+B$ has a single root in $[-1,1]$ which is in $(-1,0)$ so that

$$
\cos \phi_{0}=\left(-A+\sqrt{A^{2}-B^{2}}\right) / B
$$

Writing (5.8) as

$$
v \sin ^{2} \phi=A+B \cos \phi
$$

and then as

$$
v \cos ^{2} \phi+B \cos \phi+A-v=0,
$$

we see that $v_{0} \sin ^{2} \phi_{0}=A+B \cos \phi_{0}$. By (5.9), $\sin ^{2} \phi_{0}=2+(2 A / B) \cos \phi_{0}$, and hence

$$
v_{0}=\frac{1}{2} \frac{B^{2}}{A-\sqrt{A^{2}-B^{2}}} .
$$

Differentiating (5.11) we get

$$
\csc \phi \frac{d \phi}{d v}=-\frac{1}{B+2 v \cos \phi}
$$


and substituting for $\cos \phi$ we obtain

$$
\csc \phi \frac{d \phi}{d v}= \pm \frac{1}{\sqrt{B^{2}-4 v(A-v)}}
$$

Now the integral in (5.6) is written as $\int_{0}^{\phi_{0}}+\int_{\phi_{0}}^{\pi}$ and in $\left[0, \phi_{0}\right]$ we choose the minus sign in (5.13) and in $\left[\phi_{0}, \pi\right]$ the plus sign. We obtain

$$
q\left(x \mid y, t_{0} ; z, t_{1}\right)=\frac{e^{\lambda_{1}+\lambda_{2}}}{\pi D\left(t_{1}-t_{0}\right)} \int_{v_{0}}^{\infty} \frac{e^{-v} d v}{\sqrt{B^{2}-4 v(A-v)}}
$$

By completing the square in the radical, we get

$$
q\left(x \mid y, t_{0} ; z, t_{1}\right)=\frac{e^{\left(\lambda_{1}+\lambda_{2}\right)-\frac{A}{2}}}{\pi D\left(t_{1}-t_{0}\right)} \int_{\alpha}^{\infty} \frac{e^{-t} d t}{\sqrt{4 t^{2}-\left(A^{2}-B^{2}\right)}}
$$

with

$$
\alpha=\frac{1}{2} \sqrt{A^{2}-B^{2}}
$$

One may readily let $B=0$ in this formula. This formula is also continuous in $u_{1}$ and $u_{2}$ and one may take $u_{1}=u_{2}=1 / 2$ to obtain $q .\left(z \mid y, t_{0} ; z, t_{1}\right)$ also. The line through $y$ and $z$ corresponds to $u_{1}=u_{2}$. This does not produce any startling result as it did in the one-dimensional case.

\section{Appendix 3}

(The full formula.) In Section 3, we derived, by considering patches, the formula (3.1)

$$
p\left(x, t \mid y, t_{0} ; \omega, t_{1}\right)=\int_{-\infty}^{\infty} p\left(x, t \mid y, t_{0} ; z, t_{1}\right) d \omega(z) .
$$

By a similar consideration of patches in the variable $y$, Schrödinger [1] derives a pair of integral equations whose solvability was left open. Several proofs of the existence for special cases were given by others. The definitive existence theorem is given by Beurling [5]. We follow his formulation. Let $k(x, \xi, t)$ be the Poisson-Green's function for the problem on $\Omega$

$$
u_{t}=\Delta u, \quad u(x, 0)=f_{0}(x), \quad \frac{\partial u}{\partial n}(x, t)=0 \text { on } \partial \Omega
$$

where $f_{0}$ is the observed density at $t=0$. If the density $f_{1}(x)$ is observed at $t_{1}$ and it is not $u\left(x, t_{1}\right)$, then the most probable density is of the form 
$w(x, t)=u_{0}(x, t) u_{1}(x, t) \quad\left(0<t<t_{1}\right)$ where $u_{0}$ is a non-negative solution of

$$
u_{t}=\Delta u \text { in } \Omega, \quad \frac{\partial u}{\partial n}=0 \text { on } \partial \Omega, \quad t>0,
$$

and $u_{1}$ is a non-negative solution of the adjoint equation

$$
-u_{t}=\Delta u \text { in } \Omega, \quad \frac{\partial u}{\partial n}=0 \text { on } \partial \Omega, \quad t<t_{1} .
$$

If $g_{0}(x)=u_{0}(x, 0) \geq 0$ and $g_{1}(x)=u_{1}\left(x, t_{1}\right) \geq 0$ then

$$
u_{0}(x, t)=\int_{\Omega} k(x, \xi, t) g_{0}(\xi) d \xi, \quad t>0,
$$

and

$$
u_{1}(x, t)=\int_{\Omega} k\left(\xi, x, t_{1}-t\right) g_{1}(\xi) d \xi, \quad t<t_{1}
$$

and so

$$
\left\{\begin{array}{l}
g_{0}(x) \int_{\Omega} k\left(\xi, x, t_{1}\right) g_{1}(\xi) d \xi=f_{0}(x), \\
g_{1}(x) \int_{\Omega} k\left(\xi, x, t_{1}\right) g_{0}(\xi) d \xi=f_{1}(x)
\end{array}\right.
$$

is the system that needs to be solved for $g_{0}$ and $g_{1}$.

A. O. Barut in his review [6] of the collected works of Schrödinger remarked that this idea of Schrödinger has only recently again caught the attention of mathematicians. A specific example is the paper by Zambrini [7]. Interested readers can find references in this paper. In the case $f_{1}$ is a point mass, the formulae here agree with (6.1).

\section{Appendix 4}

We consider the center of mass of the probability distributions $p\left(x, t \mid \sigma, t_{0} ; \omega, t_{1}\right)$. We first consider $p\left(x, t \mid y, t_{0} ; z, t_{1}\right)$ which is

$$
\sqrt{\frac{t_{1}-t_{0}}{\left(t-t_{0}\right)\left(t_{1}-t\right)}} \frac{1}{\sqrt{4 \pi D}} e^{-I / 4 D}
$$

see (4.1) of Appendix 1. If we take $t=s t_{1}+(1-s) t_{0}$ then as in Appendix 1,

$$
p\left(x, t \mid y, t_{0} ; z, t_{1}\right)=\frac{1}{\sqrt{s(1-s) 4 \pi D\left(t_{1}-t_{0}\right)}} \exp \left(-\frac{[x-y+s(y-z)]^{2}}{4 D\left(t_{1}-t_{0}\right) s(1-s)}\right) .
$$


Thus in the integral $J=\int_{-\infty}^{\infty} x p\left(x, t \mid y, t_{0} ; z, t_{1}\right) d x$ we make the change of variables $v=x-y+s(y-z)$; we obtain

$$
\begin{aligned}
J & =\frac{y+s(z-y)}{\sqrt{s(1-s) 4 \pi D\left(t_{1}-t_{0}\right)}} \int_{-\infty}^{\infty} e^{-\frac{v^{2}}{4 D\left(t_{1}-t_{0}\right) s(1-s)}} d v \\
& =\frac{1}{\sqrt{\pi}}[y+s(z-y)] \int_{-\infty}^{\infty} e^{-w^{2}} d w=(1-s) y+s z
\end{aligned}
$$

So

$$
\begin{aligned}
M(t) & =\int_{-\infty}^{\infty} x \int_{-\infty}^{\infty} \int_{-\infty}^{\infty} p\left(x, t \mid \sigma, t_{0} ; \omega, t_{1}\right) d \sigma(y) d \omega(z) d x \\
& =\int_{-\infty}^{\infty} \int_{-\infty}^{\infty}[(1-s) y+s z] d \sigma(y) d \omega(z) \\
& =(1-s) \int_{-\infty}^{\infty} y d \sigma(y)+s \int_{-\infty}^{\infty} z d \omega(z) .
\end{aligned}
$$

In terms of $t$, this is

$$
M(t)=\frac{t_{1}-t}{t_{1}-t_{0}} \int_{-\infty}^{\infty} y d \sigma(y)+\frac{t-t_{0}}{t_{1}-t_{0}} \int_{-\infty}^{\infty} z d \omega(z) .
$$

That is, the centre of mass travels at constant speed from the centre of mass of $\sigma$ to the centre of mass of $\omega$. Schrödinger shows that this follow's from the fact that $p\left(x, t \mid y, t_{0} ; z, t_{1}\right)$ is the product of a solution of $u_{t}=D u_{x x}$ and of $-D u_{x x}=u_{t}$.

\section{Acknowledgement}

Research was supported in part by a grant from the Australian Research Council.

\section{References}

[1] E. Schrödinger, "Über die Umkehrung der Naturgesetze”, Sitzber. d. Preussischen Akademie der Wissenschaften, Berlin (1931) 144-153.

[2] W. J. Moore, Schrödinger: life and thought, (Cambridge Univ. Press, 1989).

[3] Ron Hansen, The assassination of Jesse James by the coward Robert Ford, (Alfred Knopf, New York, 1983).

[4] D. S. Mitrinović, Analytic inequalities, (New York, Springer Verlag, 1970).

[5] Arne Beurling, "An automorphism of product measures", Annal of Math. 72 (1960) 189-200.

[6] A. O. Barut, Mathematical Reviews 88 (1988) 5044-5046.

[7] J. C. Zambrini, "Euclidean quantum mechanics", Physical Review A 35 (1987) 36313649. 